\title{
ON THE SAMPLING OF SERIAL SECTIONING TECHNIQUE FOR THREE DIMENSIONAL SPACE-FILLING GRAIN STRUCTURES
}

\author{
GUOQUAN LIU AND HAIBO YU \\ University of Science and Technology Beijing, 100083 Beijing, China \\ E-mail: gq_liu@yahoo.com or G.Liu@ustb.edu.cn \\ (Accepted April 4, 2000)
}

\begin{abstract}
Serial sectioning technique provides plenty of quantitative geometric information of the microstructure analyzed, including those unavailable from stereology with one- and two-dimensional probes. This may be why it used to be and is being continuously served as one of the most common and invaluable methods to study the size and the size distribution, the topology and the distribution of topology parameters, and even the shape of three-dimensional space filling grains or cells. On the other hand, requiring tedious lab work, the method is also very time and energy consuming, most often only less than one hundred grains per sample were sampled and measured in almost all reported practice. Thus, a question is often asked: for typical microstructures in engineering materials, are so many grains or cells sampled adequate to obtain reliable results from this technique? To answer this question, experimental data of 1292 contiguous austenite grains in a low-carbon steel specimen obtained from the serial sectioning analysis are presented in this paper, which demonstrates the effect of sampling on the measurement of various parameters of grain size distribution and of the grain topology distribution. The result provides one of rules of thumb for grain stereology of similar microstructures.
\end{abstract}

Keywords: microstructure characterization, serial sectioning, 3D measurement, stereology, grain size, grain topology, steel, sampling strategy.

\section{INTRODUCTION}

Quantitative serial sectioning technique may be used to provide all of the geometric information that is available from ordinary stereological analysis. In addition, it circumvents the weaknesses inherent in those geometric models used to estimate size distribution functions and topological properties of 3D space-filling grain or cell structures. Thus, serial sectioning used to be and has been continuously used as one of the most common and valuable techniques to study the size, the topology and the shape of spacefilling grains or cells (Rhines et al., 1976; DeHoff, 1983; Liu, 1993). Some application examples and the resulting important findings can be found in the literature such as: Okazaki and Conrad (1972), Rhines et al. (1974), DeHoff and Liu (1985), Nunez and Domingo (1988), Liu and DeHoff (1990), Liu et al. (1991), Yu et al. (1998). Most often only less than one hundred grains per specimen were sampled and measured. The question is that, is such an amount of grains or cells sampled adequate for meaningful stereological analysis? To answer this question, experimental data of 1292 contiguous typical austenite grains in a low-carbon steel specimen obtained from serial sectioning analysis are presented in this paper. The results demonstrate the effect of sampling on the measurement of various parameters of the grain size distribution and of the grain topology distribution.

\section{MATERIALS AND PARAMETERS}

The experimental material used was a low-carbon steel whose chemical composition (wt \%) was: C 0.18, Si 0.29, Mn 1.14, Cr 1.06, V 0.11, Ti 0.062, B 0.0027, Al 0.031, N 0.0044, P 0.024, S 0.02. It was melted in a vacuum induction furnace, cast into an ingot of $28.5 \mathrm{~kg}$. The ingot was heated at $1250^{\circ} \mathrm{C}$ for 1 hour, forged into rods of $13 \mathrm{~mm}$ in diameter. From which a rod sample of $10 \mathrm{~mm}$ in diameter and $12 \mathrm{~mm}$ in height was further prepared, isothermally austenitized at $1050^{\circ} \mathrm{C}$ for 30 seconds, quenched and finally prepared for metallographic examination by means of standard procedures. Microscopic examination of three mutually perpendicular surfaces of the sample showed that the microstructure is essentially isotropic. A micrograph for this sample can be found elsewhere (Yu et al., 1998). 
Firstly 1292 grains were sampled with identical probabilities using 100 disectors of known thickness $(3 \mu \mathrm{m})$ as described in Liu et al. (1994). Secondly, the volume and the topology (the number of faces per grain was chosen in this paper) of each unbiased sampled grain were measured by serial sectioning analysis. Then, the following statistical quantities were calculated:

$$
\begin{gathered}
<x>=(1 / N) \sum x_{i} \\
\mu_{r}(x)=(1 / N) \sum\left(x_{i}-<x>\right)^{r} \\
\sigma(x)=\left[\mu_{2}(x)\right]^{1 / 2} \\
C V_{N}(x)=\sigma(x) /<x> \\
C S_{N}(x)=\mu_{3}(x) / \sigma^{3}(x) \\
C E_{N}(x)=\left[\mu_{4}(x) / \sigma^{4}(x)\right]-3
\end{gathered}
$$

where $x$ and $x_{i}$ denote the volume, $v$, or the number of faces, $f$, of individual grains and that of the $i^{\text {th }}$ sampled grain, respectively; $N$ is the total number of sampled grains; $\langle x\rangle$ is the arithmetic mean of $x, \mu_{r}$ $(x)$ is the $r^{\text {th }}$ central moment $(r=1,2,3,4)$, with $\sigma(\mathrm{x})$, $C V_{N}(x), C S_{N}(x)$ and $C E_{N}(x)$ being the standard deviation, the coefficient of variation, the skewness and the kurtosis of a given distribution of $x$, respectively.

\section{RESULTS AND DISCUSSION}

The statistical results on the volume and topological parameters of the grain structure in the specimen analyzed are as follows when the total number of sampled grains reaches to 1292 :

$$
\begin{aligned}
<v>=6.78 \times 10^{-14} \mathrm{~m}^{3}, C V_{N}(v)=1.13, & C S_{N}(v)=2.296, \\
C E_{N}(v) & =7.465 ; \\
<f>=12.91, \quad C V_{N}(f)=0.54, C S_{N}(f) & =0.08, \\
C E_{N}(f) & =0.58 .
\end{aligned}
$$

Figs. 1(a) (d) show the variation of the mean grain volume, the coefficient of variation, the skewness and the kurtosis of grain volume distribution obtained with the number of grains already sampled. It is obvious that the fluctuations are very large when $\mathrm{N}$ is not large enough. It is easy to conclude from Fig. 1 that the minimum number of grains sampled cannot be less than 200 for a reliable measurement of mean grain volume, and about 700 for the measurement of the other three volume distribution parameters in order that the relative error is smaller than $5 \%$.

The corresponding experimental results of the topological parameters for the same sample are shown in Figs. 2(a) (d). The similar conclusion may be obtained. If the mean value of the number of grain faces needs to be measured, the minimum number of sampled grains is also about 200, so is for the coefficient of variation. On the other hand, analyses of even more than 1000 grains seems inadequate for reliable evaluation of the skewness or the kurtosis of distribution of the number of grain faces.

Of course, the above conclusions from a special specimen of a special material are not necessarily versatile, because different grain shapes, grain topology and grain size distributions may appear in different materials, or even in different specimens of the same material. However, the microstructure studied here is very typical for those controlled by surface tension equilibrium, with relative uniform grain (or cell) size with isotropic grain (or cell) shape. If the microstructure concerned is more nonuniform, of course, a larger number of grains or cells should be sampled and analyzed.

Therefore, those results from experiments with less than 200 grains per sample measured should be used with special caution. Those experimental reports did provide valuable information of the size and topology of individual grains or cells, inspiring many bright ideas and further research in literature. However, they might be unreliable to be used to extract quantitative information about volume distributions or topology distributions.

The present conclusions may also be applicable to the measurement of other $3 \mathrm{D}$ size and topological parameters besides the volume and number of faces of grain, according to approximate or exact relationships among different distribution descriptors (DeHoff and Liu, 1985; Liu et al., 1994; Yu et al., 1998).

\section{CONCLUSIONS}

The effect of the number of sampled grains on the measurement of 3D grain volume and topological parameters by the serial sectioning technique was experimentally studied, using a typical 3D spacefilling uniform microstructure in materials science and engineering.

The results show that those results in literature from experiments with less than 200 sampled grains per specimen should be used with special caution, if one needs to know quantitative information of mean volume and mean number of faces in three dimensional space-filling grain or cell structures. 
It is also shown that, it is much more difficult to obtain reliable information on the coefficient of
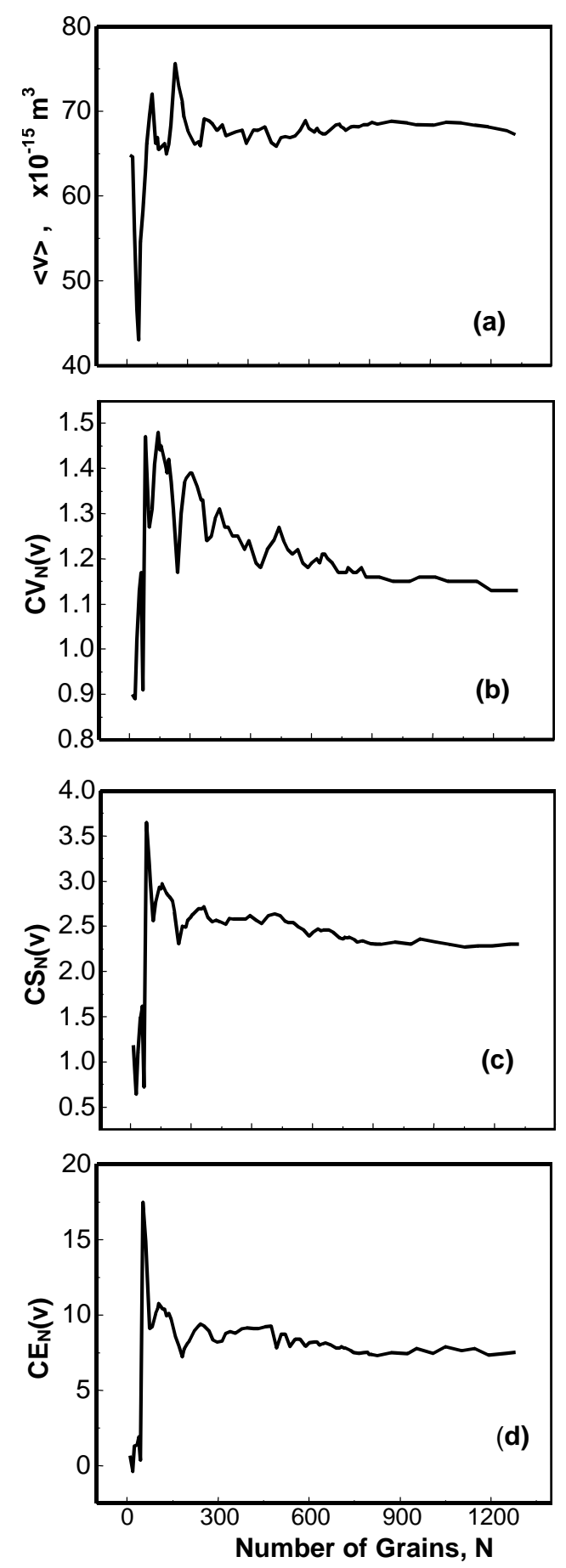

Fig. 1. Variation of selected parameters of the measured grain volume distribution with the number of grains already sampled and measured in a steel specimen. variation of grain volume, and/or on both the skewness and the kurtosis of the number of grain faces.
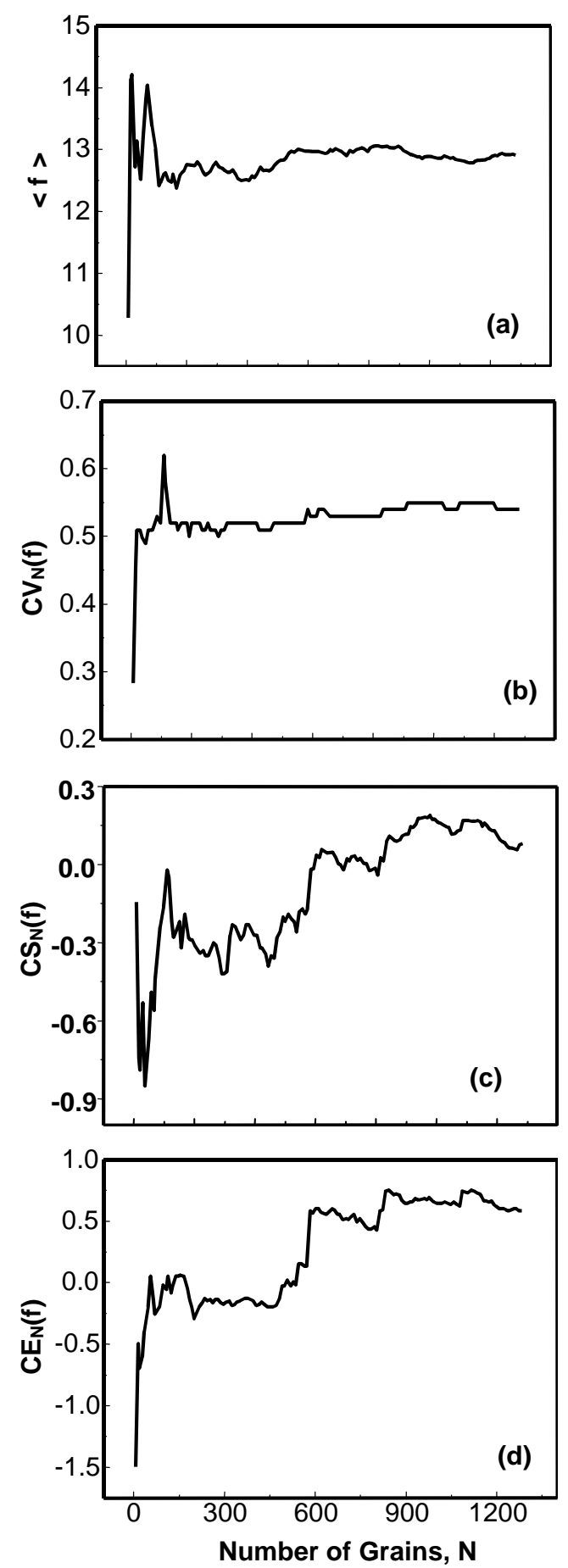

Fig. 2. Variation of selected parameters of the measured number distribution of grain faces with the number of grains sampled in the same specimen as in Fig. 1. 
A preliminary report of some of the data was presented at the Xth International Congress for Stereology, Melbourne, Australia, 1-4 November 1999.

\section{ACKNOWLEDGEMENT}

This work was supported by research grants from the National Natural Science Foundation of China (Projects 59872003, 59771070, and 59271063) and grants from the Ministry of Education of China.

\section{REFERENCES}

DeHoff RT (1983). Quantitative serial sectioning analysis: preview. J Microsc 131(3):259-63.

DeHoff RT, Liu GQ (1985). On the relation between grain size and grain topology. Metall Trans 16A:2007-11.

Liu G (1993). Invited review: Applied stereology in materials science and engineering. J Microsc 171(1):57-68.

Liu G, DeHoff RT (1990). Stereological characterization of microstructural evolution at late stages of Sintering for nickel powder compacts. Acta Metall Sinica (Series B) 3(2):115-24.
Liu G, Li W, Hu Y (1991). Saturation of preferential sites for nucleation and growth of ferrite phase at early stages of austenite decomposition in low-carbon boron steels. Acta Metall Sinica (Series A) 4(5):303-9.

Liu G, Yu H, Li W (1994). Efficient and unbiased evaluation of grain size and topology. Acta Stereol 13:281-6.

Nunez C, Domingo F (1988). Grain shape and its influence on the experimental measurement of grain size. Metall Trans 19A:933-40.

Okazaki B, Conrad H (1972). Grain size distribution in recrystallized alpha-titanium. Trans JIM 13:198-204.

Rhines FN, Craig KR, DeHoff RT (1974). Mechanism of steady-state grain growth in aluminum. Metall Trans 5A:413-25.

Rhines FN, Craig KR, Rousse DA (1976). Measurements of average grain volume and certain topological parameters by serial section analysis. Metall Trans 7A:1729-34.

Yu H, Liu G, Song X (1998). On the relation between the number-weighted and volume-weighted grain volume distribution parameters. Metall Mater Trans A 29A(12):3081-6. 